\title{
The association between triglycerides/high-density lipoprotein cholesterol ratio, insulin resistance and serum androgen levels in patients with polycystic ovary syndrome
}

\author{
Firdevs Ulutaş ${ }^{1} \oplus$, Soner Cander ${ }^{2} \oplus$, Özen Öz Gül²® \\ ${ }^{1}$ Department of Rheumatology, Pamukkale University School of Medicine, Denizli, Turkey \\ ${ }^{2}$ Department of Endocrinology and Metabolism, Uludağ University School of Medicine, Bursa, Turkey
}

\begin{abstract}
Objectives: Insulin resistance and dyslipidemia are common comorbidities of polycystic ovary syndrome. We aim to evaluate the association between triglycerides/high-density lipoprotein cholesterol ratio, insulin resistance, and serum androgen levels in patients with polycystic ovary syndrome.

Methods: We enrolled a total of 40 polycystic ovary syndrome patients and 20 healthy control subjects in this cross-sectional study. The polycystic ovary syndrome patients were divided into two subgroups obese and nonobese. The triglycerides/high-density lipoprotein cholesterol ratio and homeostatic model assessment of insulin resistance were calculated for all individuals. Demographic characteristics, serum levels of metabolic parameters, and androgens were compared between the study subgroups. $P$ - value $<0.05$ was accepted as statistically significant.

Results: The triglycerides/high-density lipoprotein cholesterol ratio was higher in obese polycystic ovary syndrome patients than in other groups $(3.64 \pm 3.06$ vs. $1.07 \pm 0.36$ in control and $1.18 \pm 0.53$ in non-obese group, $p<0.005)$. The triglycerides/high-density lipoprotein cholesterol ratio was positively correlated with homeostatic model assessment of insulin resistance $(\mathrm{r}=0.546, p<0.001)$, total testosterone $(\mathrm{r}=0.402, p=$ $0.010)$, and free androgen index $(\mathrm{r}=0.609, p<0.001)$ while was negatively correlated with sex hormonebinding globulin $(r=-0.497, p=0.001)$. Obese polycystic ovary syndrome patients had higher serum total testosterone levels, higher free androgen index, and lower sex hormone-binding globulin than non-obese polycystic ovary syndrome patients $(0.71 \pm 0.49 \mathrm{ng} / \mathrm{mL}$ vs. $0.45 \pm 0.16 \mathrm{ng} / \mathrm{mL}, p=0.006 ; 3.1 \pm 1.91$ vs. 1.01 $\pm 0.49, p<0.005$; and $26 \pm 10.3 \mathrm{nmol} / \mathrm{L}$ vs. $59.6 \pm 43.7 \mathrm{nmol} / \mathrm{L}, p<0.005$, respectively). Obese polycystic ovary syndrome patients had also worse lipid parameters, including high triglycerides and low high-density cholesterol when compared with other groups.

Conclusions: The triglycerides/high-density lipoprotein cholesterol ratio was correlated with the homeostatic model assessment of insulin resistance, and androgenic hormonal profiles including total testosterone and free androgen index in patients with polycystic ovary syndrome.
\end{abstract}

Keywords: Insulin resistance, TG/HDL-C ratio, hyperandrogenism, obesity, polycystic ovary syndrome

Dolycystic Ovary Syndrome (PCOS) is a common metabolic disease. The Rotterdam criteria includ-

ing clinical and/or biochemical findings of hyperandrogenism, oligomenorrhea or oligo-ovulation, and/or 
existence of polycystic ovaries on ultrasound are commonly used to diagnose patients with PCOS. The presence of at least two criteria is essential for the diagnosis [1].

Patients with PCOS have an increased risk for the development of type 2 diabetes mellitus (T2DM) and cardiovascular events due to concurrent insulin resistance (IR). Also, dyslipidemia and metabolic syndrome are more often seen in PCOS patients with IR than those without IR [2]. IR has been detected in most patients with PCOS beyond that predicted by their body mass index (BMI). In contrast, PCOS per se has been shown to confer a risk of IR [3]. Although definitive pathogenic mechanisms are currently not clear, IR and hyperinsulinism play a contributory role in the pathogenesis of hyperandrogenism [4]. An expert panel by clinicians recommended screening glucose intolerance in PCOS patients [5]. In the light of all this information, investigation of insulin resistance in addition to screening fasting lipid profiles, measurement of blood pressure, and determination of body mass index (BMI) may contribute to the determination of initial cardiovascular risk in these patients. Currently, there are no validated methods for indicating IR in daily practice. There are several complicated, expensive, and timeconsuming diagnostic tests and methods such as the euglycaemic hyperinsulinaemic clamp technique. Homeostatic model assessment of insulin resistance (HOMA-IR) has a lower cost and is more practical [6]. Practical measurements that evaluate simultaneously IR and cardiovascular risk are essential for PCOS patients. Hyperlipidemia related to IR presents with high levels of triglycerides (TG) and low-density lipoprotein cholesterol (LDL-C) in addition to lower highdensity lipoprotein cholesterol (HDL-C) levels. Nur Zati Iwani et al. [7] showed a great sensitivity of the TG/HDL-C ratio in diagnosing metabolic syndrome and in the determination of IR and cardiometabolic risk in obese children. Although few studies suggested the association between the TG/HDL-C ratio and IR in PCOS patients, the knowledge does not gain yet a clear recommendation for clinical practice [8]. Therefore, we aimed to evaluate TG/HDL-C ratio in Turkish PCOS patients as a predictor of IR, and its relationship with serum androgen levels. We hypothesized to obtain a practical formula from lipid parameters that are available in daily practice.

\section{METHODS}

We enrolled a total of 40 PCOS patients who fulfilled the 2004 revised Rotterdam Criteria in this cross-sectional study [10]. The PCOS patients were divided into two subgroups obese and non-obese. Patients with at least two of the subsequent criteria were accepted as PCOS; (i) oligo- or anovulation, (ii) clinical and/or biochemical hyperandrogenism, (iii) polycystic ovaries. Polycystic ovarian morphology was confirmed via ultrasound. Twenty healthy subjects with no symptoms of hirsutism, oligo-anovulation, or infertility were enrolled in the control group. Serum androgen levels were normal, and there were no pathological hyperandrogenemia signs on physical examination. The control group was selected from individuals examined by the same physician in the same time frame, not affecting the examination, especially the Ferriman-Gallwey Score (FGS) [11]. The non-obese PCOS patients and healthy controls were matched in terms of anthropometric measurements such as BMI. The detailed medical history (menstrual order, infertility, hirsutism) and systemic examination notes of all patients were examined. The exclusion criteria were also set as being pregnant and/or postmenopausal woman, having an incomplete diagnosis, using hormone preparations such as oral contraceptive drugs within the last six months, or having any concomitant confounding endocrinological diseases such as hypothyroidism, T2DM, and dyslipidemia, or systemic disorders such as coronary artery disease, renal or hepatic insufficiency or tumors of the pituitary, ovary or adrenal glands. The study was performed by following the local Ethics Committee approval and the Helsinki Declaration. All participants approved a written- and informed- consent form.

For physical measurements, height, weight, and BMI were measured. The waist circumference (WC), hip circumference (HC), and waist-to-hip ratio (WHR) were calculated for anthropometric measurements. Bodyweight was measured on a scale without shoes and extra clothing. BMI was calculated as the bodyweight (in kilograms) divided by the height (in meters) squared. BMI of at least $30 \mathrm{~kg} / \mathrm{m}^{2}$ was determined as the cut-off for obesity [9]. The midpoint between the lower border of the rib cage and the iliac crest was determined for the measurement of the waist circumference. For the hip circumference, the maximum 
diameter over the buttocks was measured with a measuring tape according to the WHO Guidelines [12, 13]. Blood samples for levels of serum gonadotropins were taken from the patients between the second and fourth days of the menstrual cycle between 08 a.m. and 10 a.m. For amenorrheic patients with PCOS, they were prescribed oral medroxyprogesterone acetate $5 \mathrm{mg}$, once a day for five days. After the initiation of the menstrual cycle, the blood samples were taken as above-mentioned. Blood samples that investigate serum levels of glucose, insulin, and lipid profile were also collected after eight-hour overnight fasting. TG/HDL-C and HOMA-IR were calculated for all individuals. The following formula 'fasting glucose $(\mathrm{mg} / \mathrm{dL}) \times$ fasting insulin $(\mu \mathrm{U} / \mathrm{mL}) / 405$ ' was used to calculate HOMA-IR [14].

\section{Statistical Analysis}

Data obtained in the study were analyzed statistically using SPSS version 21.0 (SPSS Inc.) software. Categorical variables were compared with the Chisquare test. The Student's t-test and One-way ANOVA and Tukey post hoc HSD tests were used for the analysis of independent, normally distributed parametric variables, and the Mann-Whitney U test with Bonferroni correction and the Kruskal-Wallis test were used for the analysis of numerical variables not normally distributed. Spearman's correlation coefficients were calculated to assess the associations between variables. Data were expressed as mean \pm standard devia- tion (SD), median, minimum and maximum values or number $(\mathrm{n})$, and percentage $(\%)$ as appropriate. A value of $p<0.05$ was considered statistically significant. In correlation comparisons where each group was evaluated separately, it was evaluated with Bonferroni correction $(p=0.05 / 3=0.0167)$.

\section{RESULTS}

The mean age was $26.7 \pm 7.5$ years among 40 patients with PCOS while $28.1 \pm 4.4$ years among healthy controls. The demographic and anthropometric measurements of all the study subjects are shown in Table 1. There was no difference between the healthy group and the non-obese patient groups in terms of height, weight, WC, and BMI. Hirsutism was more prominent in PCOS patients compared to the healthy control subjects, and there was no difference between the obese and non-obese PCOS groups [FGS: $15.8 \pm 6.61$ (in non-obese PCOS), FGS: $17.30 \pm 4.92$ (in obese PCOS); $p>0.05$ ]. Comparisons were made of hormonal parameters. The serum androgen levels are given in Table 2. Obese PCOS patients had higher serum total testosterone levels, higher free androgen index, and lower sex hormone-binding globulin than non-obese PCOS patients $(0.71 \pm 0.49 \mathrm{ng} / \mathrm{mL}$ vs. 0.45 $\pm 0.16 \mathrm{ng} / \mathrm{mL}, p=0.006 ; 3.1 \pm 1.91$ vs. $1.01 \pm 0.49, p$ $<0.005$; and $26 \pm 10.3 \mathrm{nmol} / \mathrm{L}$ vs. $59.6 \pm 43.7 \mathrm{nmol} / \mathrm{L}$, $p<0.005$, respectively). Non-obese PCOS patients

\section{Table 1. Comparisons of demographic and anthropometric measurements of patients according} to the groups

\begin{tabular}{lcccc}
\hline & $\begin{array}{c}\text { Control group } \\
(\mathbf{n}=\mathbf{2 0})\end{array}$ & $\begin{array}{c}\text { Non-obese PCOS group } \\
(\mathbf{n}=\mathbf{2 0})\end{array}$ & $\begin{array}{c}\text { Obese PCOS group } \\
(\mathbf{n = 2 0})\end{array}$ & $p$ value \\
\hline Age $($ years $)$ & $28.10 \pm 4.48$ & $23.10 \pm 5.60$ & $30.40 \pm 7.60$ & $<0.005^{1,3}$ \\
Height $(\mathrm{cm})$ & $161.10 \pm 7.67$ & $163.20 \pm 5.25$ & $161.40 \pm 6.31$ & $0.038^{2}$ \\
\hline Weight $(\mathrm{kg})$ & $61.40 \pm 7.72$ & $58.60 \pm 8.17$ & $95.00 \pm 12.35$ & $<0.005^{2,3}$ \\
BMI $\left(\mathrm{kg} / \mathrm{m}^{2}\right)$ & $21.80 \pm 2.64$ & $21.70 \pm 3.04$ & $36.10 \pm 4.24$ & $<0.005^{2,3}$ \\
WC $(\mathrm{cm})$ & $74.10 \pm 5.43$ & $71.00 \pm 4.97$ & $99.50 \pm 10.30$ & $<0.005^{2,3}$ \\
WHR & $0.77 \pm 0.03$ & $0.75 \pm 0.02$ & $0.84 \pm 0.04$ & $<0.05^{1,2,3}$ \\
Ferriman Gallwey Score & $2.85 \pm 1.18$ & $15.8 \pm 6.61$ & $17.30 \pm 4.92$ & $<0.005^{1,2}$ \\
\hline
\end{tabular}

Data are shown as mean \pm standard deviation. PCOS $=$ polycystic ovary syndrome, $\mathrm{BMI}=$ body mass index, WC $=$ waist circumference, $\mathrm{WHR}=$ waist-hip ratio, $\mathrm{FGS}=$ Ferriman Gallwey score

$p^{1}=$ Comparison of the control group and non-obese PCOS group

$p^{2}=$ Comparison of the control group and obese PCOS group

$p^{3}=$ Comparison of obese and non-obese PCOS groups 
Table 2. Comparisons of hormonal and biochemical data of the patients according to the groups

\begin{tabular}{|c|c|c|c|c|}
\hline & $\begin{array}{c}\text { Control } \\
\text { group } \\
(n=20)\end{array}$ & $\begin{array}{l}\text { Non-obese } \\
\text { PCOS group } \\
\quad(\mathbf{n}=\mathbf{2 0})\end{array}$ & $\begin{array}{c}\text { Obese PCOS } \\
\text { group } \\
(\mathbf{n}=\mathbf{2 0})\end{array}$ & $p$ value \\
\hline Total testosterone (ng/mL) & $0.29 \pm 0.08$ & $0.45 \pm 0.16$ & $0.71 \pm 0.49$ & $<0.005^{1,2,3}$ \\
\hline $\begin{array}{l}\text { Sex hormone binding globülin } \\
\text { (nmol/L) }\end{array}$ & $55.20 \pm 31.10$ & $59.60 \pm 43.70$ & $26.00 \pm 10.30$ & $<0.005^{2,3}$ \\
\hline Free androgen index & $0.62 \pm 0.28$ & $1.01 \pm 0.49$ & $3.10 \pm 1.91$ & $<0.05^{1,2,3}$ \\
\hline Androstenedione (ng/mL) & $1.96 \pm 0.64$ & $3.73 \pm 1.41$ & $4.20 \pm 1.71$ & $<0.005^{1,2}$ \\
\hline $\begin{array}{l}\text { Dehydroepiandrosterone-sulfate } \\
(\mu \mathrm{g} / \mathrm{dL})\end{array}$ & $\begin{array}{c}261.40 \pm \\
111.70\end{array}$ & $360.40 \pm 143.70$ & $348.40 \pm 107.00$ & $<0.005^{1,2}$ \\
\hline $\begin{array}{l}\text { Follicular stimulating hormone } \\
(\mathrm{mIU} / \mathrm{mL})\end{array}$ & $5.98 \pm 5.36$ & $4.28 \pm 3.61$ & $6.62 \pm 6.95$ & $0.049^{3}$ \\
\hline Luteinizing hormone (mIU/mL) & $6.25 \pm 7.90$ & $9.57 \pm 18.61$ & $8.20 \pm 5.30$ & $\mathbf{0 . 0 3}^{2}$ \\
\hline Fasting plasma glucose $(\mathrm{mg} / \mathrm{dL})$ & $71.60 \pm 12.55$ & $83.30 \pm 4.69$ & $88.30 \pm 8.29$ & $<0.05^{1,2,3}$ \\
\hline Fasting plasma insulin (mIU/L) & $7.06 \pm 2.39$ & $8.01 \pm 1.57$ & $16.60 \pm 7.65$ & $<0.005^{2,3}$ \\
\hline Aspartate transaminase (IU/L) & $17.20 \pm 2.30$ & $16.40 \pm 3.20$ & $18.30 \pm 4.90$ & 0.723 \\
\hline Alanine transaminase (IU/L) & $14.30 \pm 6.50$ & $12.70 \pm 6.70$ & $21.80 \pm 8.20$ & $<0.005^{2,3}$ \\
\hline Triglyceride (mg/dL) & $64.60 \pm 20.80$ & $67.40 \pm 25.70$ & $150.20 \pm 99.70$ & $<0.005^{2,3}$ \\
\hline $\begin{array}{l}\text { Low-density lipoprotein cholesterol } \\
\text { (mg/dL) }\end{array}$ & $95.40 \pm 20.30$ & $103.00 \pm 28.30$ & $129.50 \pm 36.90$ & $<\mathbf{0 . 0 5}^{2,3}$ \\
\hline $\begin{array}{l}\text { High-density lipoprotein cholesterol } \\
\text { (mg/dL) }\end{array}$ & $60.50 \pm 9.70$ & $58.80 \pm 11.10$ & $44.10 \pm 9.70$ & $<0.005^{2,3}$ \\
\hline TG/HDL-C & $1.07 \pm 0.36$ & $1.18 \pm 0.53$ & $3.64 \pm 3.06$ & $<0.005^{2,3}$ \\
\hline HOMA-IR & $1.24 \pm 0.49$ & $1.62 \pm 0.35$ & $3.59 \pm 1.62$ & $<0.005^{1,2,3}$ \\
\hline
\end{tabular}

PCOS = polycystic ovary syndrome, TG/HDL-C ratio = triglycerides: high-density lipoprotein-cholesterol ratio, HOMA-IR

$=$ homeostatic model assessment insulin resistance

$p^{1}=$ Comparison of control group and non-obese PCOS group

$p^{2}=$ Comparison of control group and obese PCOS group

$p^{3}=$ Comparison of obese and non-obese PCOS groups

had higher free androgen index, androstenedione and DHEAS levels than the healthy control group $(1.01 \pm$ $0.49, \mathrm{p}=0.012 ; 3.73 \pm 1.41, p<0.005 ; 360.4 \pm 143.7$, $p=0.028)$.

When the groups were evaluated in terms of laboratory data, LDL-C and triglyceride levels were significantly higher, and HDL-C levels were significantly lower in the obese PCOS group when compared to the other two groups (Table 2). While there were no differences in aspartate transaminase (AST) levels between groups, serum ALT levels were higher in the obese PCOS group than in the other two groups. (21.8 $\pm 8.2 \mathrm{IU} / \mathrm{L}, p<0.005)$. The TG: HDL-C ratio and HOMA-IR values were significantly higher in obese patients with PCOS than non-obese PCOS patients
( $3.64 \pm 3.06$ vs. $1.18 \pm 0.53, p<0.005$ and $3.59 \pm 1.62$ vs. $1.62 \pm 0.35, \mathrm{p}<0.005$; respectively).

In addition, non-obese PCOS patients had significantly higher serum fasting glucose and HOMA-IR than healthy individuals $(83.3 \pm 4.69, p<0.005 ; 1.62$ $\pm 0.35, p<0.005$, respectively). When all the patients were evaluated, according to the results of correlation analysis, the TG/HDL-C ratio was positively correlated with HOMA-IR $(\mathrm{r}=0.546, p<0.001)$, total testosterone $(r=0.402, p=0.010)$, and FAI $(r=0.609$, $p<0.001)$ while was negatively correlated with sex hormone-binding globulin $(\mathrm{r}=-0.497, p=0.001)$ (Table 3). We detected no significant correlation when each group was separately examined. 
Table 3. Correlation between hormonal parameters and the TG/HDL-C ratio in the patients

\begin{tabular}{lcccccccc}
\hline & $\begin{array}{c}\text { All obese and } \\
\text { non-obese } \\
\text { patients with } \\
\text { PCOS }\end{array}$ & Obese PCOS & Non-obese PCOS & Healthy controls \\
& $\mathbf{r}$ & $\boldsymbol{p}$ value & $\mathbf{r}$ & $\boldsymbol{p}$ value & $\mathbf{r}$ & $\boldsymbol{p}$ value & $\mathbf{r}$ & $\boldsymbol{p}$ value \\
\hline TG/HDL-C & 0.546 & $<\mathbf{0 . 0 0 1}$ & 0.064 & 0.787 & 0.099 & 0.676 & 0.193 & 0.416 \\
\hline HOMA-IR & 0.402 & $\mathbf{0 . 0 1 0}$ & 0.098 & 0.682 & 0.148 & 0.533 & 0.077 & 0.746 \\
\hline Total testosterone & -0.497 & $\mathbf{0 . 0 0 1}$ & -0.046 & 0.846 & -0.207 & 0.381 & 0.226 & 0.338 \\
\hline $\begin{array}{l}\text { Sex hormone-binding } \\
\text { globulin }\end{array}$ & 0.609 & $<\mathbf{0 . 0 0 1}$ & 0.059 & 0.806 & 0.344 & 0.138 & -0.071 & 0.767 \\
Free androgen index & 0.161 & 0.321 & -0.129 & 0.589 & 0.461 & 0.041 & -0.015 & 0.950 \\
\hline Dehydroepiandrosterone & 0.122 & 0.452 & 0.091 & 0.703 & -0.081 & 0.735 & -0.318 & 0.172 \\
\hline Androstenedione & & & & & & & & \\
\hline
\end{tabular}

HOMA-IR = homeostatic model assessment insulin resistance, TG/HDL-C ratio = triglycerides: high-density lipoproteincholesterol ratio. Spearman's rho correlation coefficient with Bonferroni correction $(p=0.05 / 3=0.0167)$.

\section{DISCUSSION}

Obesity and IR are well-known risk factors contributing to the complex pathophysiological mechanisms in PCOS. A vast majority of patients with PCOS (40-88\%) are overweight/obese and insulin-resistant [15]. IR is an insufficient glucose response to insulin and is often diagnosed with clinical manifestations, including T2DM and metabolic syndrome [16]. There is a need for a reliable, practical, valid parameter that can indicate IR. HOMA-IR is a commonly used tool for IR. The analysis of serum fasting insulin is not possible and practical in every health center to calculate HOMA-IR. Also, no clear cut-off value has been established for HOMA-IR. The euglycemic clamp technique is another reported method performed in specialized centers but is also impractical for routine use [17]. Both of the-mentioned methods have not been placed for routine clinical use in detecting IR because of limitations related to cost, reliability and standardization. Mc Laughlin et al. [18] showed that a TG/HDL-C ratio of at least 3 was more diagnostic than TG or insulin levels alone in determining IR in overweight patients. The number of 1.8 in SI units (3.0 in traditional units) was reported as a cut-off point for TG/HDL-C (with a sensitivity and specificity; 57\%, and $71 \%$ respectively). Song do et al. [19] recommended OGTT for Korean women with PCOS with TG/HDL-C ratio $>2.5$, and this cut-off was calculated as a predictor of IR. Greenwood and Huddleston [20] showed that the cut-off value of 2.64 had a sensitivity of $87 \%$ and a specificity of $75 \%$ for diagnosing metabolic syndrome in PCOS patients. The mean TG/HDL-C ratio was 3.64 in obese PCOS patients in our study. We noticed again that the TG/HDL-C ratio was positively correlated with HOMA-IR in our population despite this correlation may be explained by the presence of obesity alone. The TG/HDL-C ratio was similar between non-obese PCOS patients and healthy controls. These results may have been also affected by the low patient numbers in each group and the patient characteristics.

The average BMI, WC, and WHR, were similar in the non-obese PCOS patient and the healthy control groups. Ovesen et al. [21] found no difference in IR in non-obese PCOS patients compared to an age and weight-matched control group. Although the TG/HDL-C ratio was similar between the two subgroups, non-obese PCOS patients had higher HOMAIR values than the control group without a statistical significance. It is possible to say that HOMA-IR can detect IR earlier than the TG/HDL-C. This condition may be explained by increased levels of serum fasting insulin before dyslipidemia occurs.

There are many complex pathogenetic pathways in the underlying mechanism of hyperandrogenism and IR. Higher levels of different androgens may contribute to IR or vice versa. It is well-known that patients with hyperinsulinemia have marked hyperandrogenemia [22]. Excess insulin levels reduce hepatic sex hormone-binding globulin and increase ovarian androgen production via stimulation of ovar- 
ian insulin growth factor-1 receptors in the presence of the adequate luteinizing hormone [23]. As a result, IR and obesity aggravate hyperandrogenism's clinical and laboratory findings, and losing weight reduces androgen levels in patients [24]. Nevertheless, hyperandrogenemia has a negligible effect on IR [25]. Holte et al. [26] reported that the body fat ratio had been shown to worsen hyperandrogenism findings in women with PCOS, regardless of BMI. According to that cross-sectional study, obese PCOS patients with a higher body fat ratio also have a higher free androgen index, higher total testosterone, and lower SHBG [26]. The conclusion drawn from our study is also that obese PCOS patients had higher total testosterone levels, higher FGS, and higher TG/HDL-C ratio values than non-obese PCOS patients and healthy control subjects.TG/HDL-C ratio was positively correlated with serum androgen levels and free androgen index, and negatively correlated with SHBG. Previous studies have indicated that SHBG can be used as a marker of IR [27]. The SHBG values were lower in obese PCOS patients than in the other groups. This condition might be a causative factor for more severe hirsutism and hyperandrogenism findings. Ain et al. [28] also investigated the TG/HDL-C ratio in a study of 350 participants without comorbidity or chronic illness. The TG/HDL-C ratio was correlated with carotid intimal thickness. They noticed that TG/HDL-C ratio can be used as an early predictor of cardiometabolic events. TG/HDL-C ratio was also shown as an indicator of atherosclerotic risk, even in chronic inflammatory diseases such as ankylosing spondylitis and familial Mediterranean fever [29].

\section{Limitations}

The limitations of the study were as follows: A major limitation of the study was the evaluation of IR with HOMA-IR. Secondly, the sample size was limited. More patients are needed to determine a specific cut-off value for the TG/HDL-C ratio and compare non-obese PCOS and healthy control subjects. Another limitation was that it was a cross-sectional study in which causal relationships cannot be evaluated. However, determination of the TG/HDL-C ratio in patients with PCOS can be used as a good predictor as a surrogate marker for IR and cardiometabolic risk. The assessment of the FGS was performed only by one of the researchers at one time was another limitation pre- venting determining intra- and inter-observer variations.

\section{CONCLUSION}

This study demonstrated a higher TG/HDL-C ratio and IR in obese PCOS patients. The TG/HDL-C ratio was also correlated with hyperandrogenism and HOMA-IR in PCOS patients. The TG/HDL-C ratio is easily calculated from the lipid parameters, and it may be practical to use TG/HDL-C ratio to predict IR in PCOS patients.

\section{Authors' Contribution}

Study Conception: ÖÖG, SC; Study Design: ÖÖG, SC, FV; Supervision: ÖÖG, SC, FV; Funding: ÖÖG, SC, FV; Materials: ÖÖG, SC, FV; Data Collection and/or Processing: ÖÖG, SC, FV; Statistical Analysis and/or Data Interpretation: ÖÖG, SC, FV; Literature Review: ÖÖG, SC; Manuscript Preparation: ÖÖG, SC, FV and Critical Review: ÖÖG, SC.

\section{Conflict of interest}

The authors disclosed no conflict of interest during the preparation or publication of this manuscript.

\section{Financing}

The authors disclosed that they did not receive any grant during conduction or writing of this study.

\section{REFERENCES}

1. Legro RS, Arslanian SA, Ehrmann DA, Hoeger KM, Murad HM, Pasquali R, et al. Diagnosis and treatment of polycystic ovary syndrome: an Endocrine Society clinical practice guideline. J Clin Endocrinol Metab 2013;98:4565-92.

2. Apridonidze T, Essah PA, Luorno MJ, Nestler JE. Prevalence and characteristics of the metabolic syndrome in women with polycystic ovary syndrome. J Clin Endocrinol Metab 2005;90:1929-35.

3. Dunaif A, Segal KR, Futterweit W, Dobrjansky A. Profound peripheral insulin resistance, independent of obesity, in polycystic ovary syndrome. Diabetes 1989;38:1165-74.

4. Dunaif A, Madeli J, Fluh H. The impact of obesity and chronic hyperinsulinemia on gonadotropin release and gonadal steroid secretion in the polycystic ovary syndrome. J Clin Endocrinol Metab 1998;66:131-9.

5. Salley KE, Wickham EP, Cheang KI, Essah PA, Karjane NW, Nestler JE. Glucose intolerance in polycystic ovary syndrome-a 
position statement of the Androgen Excess Society. J Clin Endocrinol Metab 2007;92:4546-56.

6. Holzinger U, Kitzberger R, Fuhrmann V, Funk GC, Madl C, Ratheiser K. Correlation of calculated indices of insülin resistance (QUICKI and HOMA) with the euglycaemic hyperinsulinaemic clamp technique for evaluating insülin resistance in critically ill patients. Eur J Anaesthesiol 2007;24:966-70.

7. Nur Zati Iwani AK, Jalaluddin MY, Wan Mohd Zin RM, Fuziah MZ, Hua Hong JY, Abqariyah Y, et al. TG/HDL-C ratio Is a good marker to identify children affected by obesity with increased cardiometabolic risk and insulin resistance. Int J Endocrinol 2019; 2019:8586167.

8. Ebrahimi-Mamaghani M, Saghafi-Asl M, Pirouzpanah S, Aliasgharzadeh A, Aliashrafi S, Rezayi N, et al. Association of insulin resistance with lipid profile, metabolic syndrome, and hormonal aberrations in overweight or obese women with polycystic ovary syndrome. J Health Popul Nutr 2015;33:157-67.

9. Clinical Guidelines on the Identification, Evaluation, and Treatment of Overweight and Obesity in Adults--The Evidence Report. National Institutes of Health. Obes Res 1998;6 Suppl $2: 51 \mathrm{~S}$.

10. Rotterdam ESHRE/ASRM-Sponsored PCOS Consensus Workshop Group. Revised 2003 consensus on diagnostic criteria and long-term health risks related to polycystic ovary syndrome. Fertil Steril 2004; 1:19-25.

11. Escobar-Morreale HF, Carmina E, Dewailly D, Gambineri A, Kelestimur F, Moghetti P, et al. Epidemiology, diagnosis and management of hirsutism: a consensus statement by the Androgen Excess and Polycystic Ovary Syndrome Society. Hum Reprod Update 2012;18:146-70.

12. Ross R, Neeland IJ, Yamashita S, Shai I, Seidell J, Magni P, et al. Waist circumference as a vital sign in clinical practice: a Consensus Statement from the IAS and ICCR Working Group on Visceral Obesity. Nat Rev Endocrinol 2020;16:177-89.

13. Veitch D. Where is the human waist? Definitions, manual compared to scanner measurements. Work 2012;41 Suppl 1:4018-24.

14. Legro RS, Castracane VD, Kauffman RP. Detecting insulin resistance in polycystic ovary syndrome: purposes and pitfalls. Obstetrics Gynecol Survey 2004;59:141-54.

15. Barber TM, McCarthy MI, Wass JA, Franks S. Obesity and polycystic ovary syndrome. Clin Endocrinol 2006;65:137-45.

16. Zavaroni I, Dall'Aglio E, Alpi O, Bruschi F, Bonora E, Pezzarossa A, et al. Evidence for an independent relationship between plasma insulin and concentration of high-density lipoprotein cholesterol and triglyceride. Atherosclerosis 1985;55:259-66.

17. Anderwald C, Anderwald-Stadler M, Promintzer M, Prager G, Mandl M, Nowotny P, et al. The Clamp-Like Index: a novel and highly sensitive insulin sensitivity index to calculate hyperinsulinemic clamp glucose infusion rates from oral glucose tolerance tests in nondiabetic subjects. Diabetes Care 2007;30:2374-80.

18. McLaughlin T, Abbasi F, Cheal K, Chu J, Lamendola C, Reaven G. Use of metabolic markers to identify overweight individuals who are insulin resistant. Ann Intern Med 2003;139:802-9.

19. Song do K, Lee H, Sung YA, Young Oh J. Triglycerides to high-density lipoprotein cholesterol ratio can predict impaired glucose tolerance in young women with polycystic ovary syndrome. Yonsei Med J 2016;57:1404-11.

20. Greenwood EA, Huddleston HG. Insulin resistance in polycystic ovary syndrome: concept versus cut-off. Fertil Steril 2019;112:827-8.

21. Ovesen P, Moller J, Ingerslev HJ, Jørgensen JO, Mengel A, Schmitz O, et al. Normal basal and insulin-stimulated fuel metabolism in lean women with the polycystic ovary syndrome. J Clin Endocrinol Metab 1993;77:1636-40.

22. Taylor SI, Dons RF, Hernandez E, Roth J, Gorden P. Insulin resistance associated with androgen excess in women with autoantibodies to the insulin receptor. Ann Intern Med 1982;97:8515.

23. Barbieri RL, Smith S, Ryan KJ. The role of hyperinsulinemia in the pathogenesis of ovarian hyperandrogenism. Fertil Steril 1988;50:197-212.

24. Zeng X, Xie YJ, Liu YT, Long SL, Mo ZC. Polycystic ovarian syndrome: correlation between hyperandrogenism, insulin resistance, and obesity. Clin Chim Acta 2020;502:214-21.

25. Pasquali R, Antenucci D, Casimirri F, Venturoli S, Paradisi R, Fabbri R, et al. Clinical and hormonal characteristics of obese amenorrheic hyperandrogenic women before and after weight loss. J Clin Endocrinol Metab 1989;68:173-9.

26. Holte J, Bergh T, Gennarelli G, Wide L. The independent effects of polycystic ovary syndrome and obesity on serum concentrations of gonadotrophins and sex steroids in premenopausal women. Clin Endocrinol (Oxf) 1994;41:473-81.

27. Wallace IR, McKinley MC, Bell PM, Hunter SJ. Sex hormone-binding globulin and insulin resistance. Clin Endocrinol (Oxf) 2013;78:321-9.

28. Ain QU, Asif N, Alam A, Gilani M, Shahzad N, Sheikh W. Triglycerides-to-HDL-C ratio as a marker of cardiac disease and vascular risk factors in adults. J Coll Physicians Surg Pak 2019;29:1034-7.

29. Keles N, Aksu F, Aciksari G, Yilmaz Y, Demircioglu K, Kostek O, et al. Is triglyceride/HDL ratio a reliable screening test for assessment of atherosclerotic risk in patients with chronic inflammatory disease? North Clin Istanb 2016;3:39-45. 